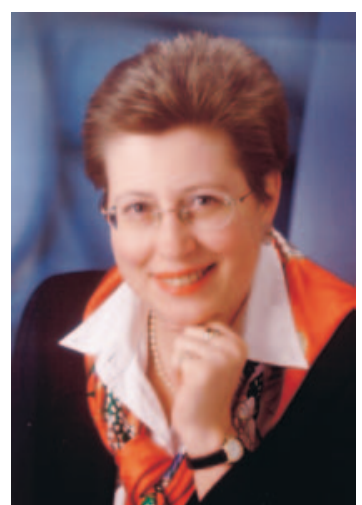

Frau Dr. Schunder-Tatzber, wie schätzen Sie die Situation der Komplementärmedizin in Österreich im Vergleich $z u$ anderen europäischen Ländern ein?

In Österreich hat die Komplementärmedizin eine andere Ausgangslage als in manch anderen europäischen Ländern, da in Österreich keine Heilpraktiker tätig sind und die meisten komplementärmedizinischen Techniken daher legal nur von Ärzten ausgeübt werden dürfen. Diese gesetzliche Grundlage hatte die Kommunikation der komplementärmedizinisch tätigen Ärzte mit der «Schulmedizin» von Anfang an erleichtert, da die universitäre Ausbildung den Anbietern dieser Techniken half, die Grenzen und möglichen Gefahren zu erkennen. Durch langjährige Aktivitäten der Wiener Internationalen Akademie für Ganzheitsmedizin, die in Kongressveranstaltungen immer die sinnvolle Verbindung darstellte und einforderte, ist es auch gelungen, einen Dialog $\mathrm{zu}$ entwickeln und feindselige Angriffe - bis hin zum Berufsverbot für Ärzte, die Komplementärmedizin anbieten, wie etwa in Schweden weitgehend $\mathrm{zu}$ vermeiden. An den Universitätskliniken gibt es nun bereits komplementärmedizinische Zusatzangebote für Patienten - beispielsweise homöopathische und Akupunkturambulanzen. Leider haben

\title{
Komplementärmedizin in Österreich
}

Frau Dr. med. Susanne Schunder-Tatzber ist als niedergelassene Ärztin für Allgemeinmedizin mit Schwerpunkt Ganzheitsmedizin, Arbeitsmedizin und Consulting in Wien tätig. Seit 1992 ist sie zudem als Mitarbeiterin an der Wiener Internationalen Akademie für Ganzheitsmedizin (Gamed; www.gamed.or.at) in den Bereichen Dokumentation, internationale Kooperationen und Ausbildung aktiv. Frau Dr. Schunder-Tatzber hat unter anderem das Studium der Medizin an der Universität Wien und ein Studium für Traditionelle Chinesische Medizin an der Hochschule für TCM, Fuzhou, China, absolviert.

wir im Gegensatz zu Deutschland, Schweiz oder England keine Lehrstühle für Komplementärmedizin an den medizinischen Hochschulen, was auch die Forschung in Österreich auf diesem Gebiet nicht begünstigt.

Die Patienten fragen diese Leistungen verstärkt nach und müssen diese aber auch privat bezahlen, da die meisten dieser Methoden nicht von den Krankenkassen erstattet werden.

Wie wichtig sind Vernetzungen und Netzwerke, wie z.B. EICCAM (European Information Center on Complementary and Alternative Medicine) oder Cambrella? Was wurde schon unternommen, was muss noch getan werden?

Der internationale Austausch wird heute immer wichtiger, um auch gemeinsam besser Anliegen auf europäischer Ebene durchsetzen zu können. Leider gibt es in Europa in einigen Ländern, aber auch in den Behörden der Europäischen Union noch immer viel Unwissenheit über komplementärmedizinische Techniken - die manchmal noch immer mit Scharlatanerie gleichgesetzt und als unwirksam und gar schädlich für Patienten dargestellt werden.

Der Verbreitung von wissenschaftlichen Erkenntnissen zum Thema
Komplementärmedizin hat sich das Netzwerk EICCAM verschrieben.

Cambrella ist das erfreuliche Ergebnis europäischer Zusammenarbeit - denn hier ist es gelungen, gemeinsam einen Forschungsschwerpunkt in Komplementärmedizin im Europäischen Forschungsrahmenprogramm FP 7 zu platzieren und nun systematisch die aktuelle Situation der Komplementärmedizin in Europa zu erfassen und Schwerpunkte für die weitere Forschung zu erarbeiten. Die Ergebnisse dieses Projektes werden dann zu weiteren Forschungsschwerpunkten führen, und an den Möglichkeiten, dass diese dann auch wieder im Europäischen Forschungsprogramm gefördert werden, muss nun gearbeitet werden, denn jetzt laufen die Vorbereitungen für das achte Rahmenprogramm.

Bitte beschreiben Sie die Rolle der Gamed, ihre Tätigkeitsfelder sowie die zukünftigen Aufgaben.

Die Gamed hat sich immer als Vermittlerin von sinnvollen Methoden der Komplementärmedizin, Einforderin von integrativen Ansätzen - im österreichischen Sprachgebrauch der Ganzheitsmedizin sowie als Mahnerin bei Fehlentwicklungen gesehen. Kongresse, Tagungen und Bücher be-

\section{KARGER}

Fax +49761 4520714 
legen diese Ausrichtung und werden in den nächsten Jahren auch weiter ein Schwerpunkt der Tätigkeit der Gamed sein und bleiben.

War die Ausrichtung der Gamed in den ersten Jahren (sie gibt es nun seit 1988) ausschliesslich auf Österreich fokussiert, haben wir in den letzten Jahren - mit der Initiative zur Ermöglichung von komplementärmedizinischen Forschungsschwerpunkten im 7. EU-Rahmenprogramm - einen etwas mehr internationalen, vor allem europäischen Aspekt entwickelt. Die Mitarbeit der Gamed im CambrellaProjekt ist ein weiterer Schritt in diese Richtung und soll auch in der Zukunft weiterentwickelt werden. Weiters sollen in der Zukunft Kompetenzen hinsichtlich der Durchführung von Forschungsprojekten entwickelt werden, um hier auch weitere Entwicklungschancen für die Gamed zu initiieren.

Welche Fortbildungsmöglichkeiten oder Programme gibt es im Bereich der Komplementärmedizin in Österreich?

Der Schwerpunkt der komplementärmedizinischen Ausbildung in Österreich liegt in der Hand von medizinischen Organisationen und Vereinigungen, die zum Teil schon seit Jahrzehnten die Ausbildung organisieren. Die Qualitätssicherung dieser Ausbildung erfolgt über die Österreichische Ärztekammer - die für Methoden wie Akupunktur, Homöopathie, Neuraltherapie oder Phytotherapie Ausbildungsstandards festgelegt hat, die diese Ausbildungsorganisationen akkreditiert hat und die nach Ausbildung und bestandener Prüfung auch die Ärztekammerdiplome verleiht.

Wie sieht die Zukunft der Komplementärmedizin in Europa aus? Wie wirkt sich Ihrer Einschätzung nach das Ja zur Komplementärmedizin in der Schweiz vom Mai 2009 darauf aus?

Solche Beispiele sind immer hilfreich, denn sie zeigen Politikern und Verantwortlichen auf, dass die Bevölkerung in einem Staat einen deutlichen Willen zur Komplementärmedizin ausgedrückt hat. Sicherlich können solche Ergebnisse nicht immer völlig auf andere europäische Länder umgelegt werden - da ja auch in der Schweiz andere legistische Rahmenbedingungen herrschen -, aber sie sind ein sichtbarer Hinweis auf den Wunsch der Bevölkerung nach Vielfalt im Behandlungsspektrum - und da unterscheiden sich die Europäer nicht so deutlich.

Die Zukunft der Komplementärmedizin in Europa wird sich vor allem auch durch eine Zunahme von Forschungsaktivitäten entwickeln, die immer mehr Hinweise auf die sinnvolle Integration dieser Methoden in den Behandlungskanon geben werden und die auch von Politikern und Versicherungen nicht mehr übersehen werden können.
Wie sind Sie mit der Komplementärmedizin in Berührung gekommen? Was bedeutet diese für Sie persönlich? Was fasziniert Sie daran besonders?

Ich hatte im Wintersemester 1988/89 die Chance, an der Hochschule für Traditionelle Chinesische Medizin ein Semester unterrichten und studieren zu können. Diese Zeit war für mich sehr prägend, hat sie mir doch eine völlig neue Sichtweise auf die Medizin eröffnet. Nach meiner Rückkehr aus China begann sich die Akademie für Ganzheitsmedizin zu entwickeln, und es war für mich sehr schön, mich hier zu engagieren.

Mich faszinieren noch immer die Vielfalt der Möglichkeiten und die Chance, dem Patienten eine andere Perspektive eröffnen - eine Verbindung von mehren sinnvollen Optionen für den Patienten anzubieten zu können. Besonders begeistert mich nun, dass wir heute neben dem traditionellen Wissen, wie der alten Chinesen oder der Homöopathie, auch immer mehr wissenschaftliche Erkenntnisse haben, dass diese Methoden nicht nur einen empirischen Hintergrund haben, sondern auch mit wissenschaftlicher Evidenz in Bezug auf Wirkmechanismen und Effekte aufwarten können.

Liebe Frau Dr. Schunder-Tatzber, herzlichen Dank für das Interview!

Interview: Alexander Eitner 